\title{
Sensing characteristics of a novel two-section long-period grating
}

\author{
Thomas Allsop, Ron Neal, Domenico Giannone, David J. Webb, Des J. Mapps, and \\ lan Bennion
}

\begin{abstract}
The behavior of a temperature self-compensating, fiber, long-period grating (LPG) device is studied. This device consists of a single 325- $\mu$ m-period LPG recorded across two sections of a single-mode B-Ge-codoped fiber-one section bare and the other coated with a 1- $\mu \mathrm{m}$ thickness of Ag. This structure generates two attenuation bands associated with the eighth and ninth cladding modes, which are spectrally close together $(\sim 60 \mathrm{~nm})$. The attenuation band associated with the Ag-coated section is unaffected by changes in the refractive index of the surrounding medium and can be used to compensate for the temperature of the bare-fiber section. The sensor has a resolution of $\pm 1.0 \times 10^{-3}$ for the refractive index and $\pm 0.3^{\circ} \mathrm{C}$ for the temperature. The effect of bending on the spectral characteristics of the two attenuation bands was found to be nonlinear, with the Ag-coated LPG having the greater sensitivity. (C) 2003 Optical Society of America
\end{abstract}

OCIS codes: $\quad 060.2370,060.2310$.

\section{Introduction}

A fiber long-period grating (LPG) is an axially periodic refractive-index variation with a period of typically a few hundred micrometers inscribed in the core of a photosensitive single-mode optical fiber by ultraviolet irradiation. Study of the LPG attenuation bands has yielded many potential applications. One important area is in fiber-optic telecommunications, where LPGs have found use as wavelength-selective filters for flattening the gain spectrum of Er-doped fiber amplifiers. ${ }^{1,2}$ Also, over the past few years, LPGs have found numerous applications in the field of sensing because of their sensitivity to strain, temperature, and the refractive index of the surrounding medium. ${ }^{3-5}$ LPG sensitivity can manifest itself in two ways; first, through the spectral shift in the attenuation band, which here we refer to as spectral sensitivity, and, second, through a change in the strength of the attenuation band.

T. Allsop (t.d.p.allsop@aston.ac.uk), D. Giannone, D. J. Webb, and I. Bennion are with the Photonics Research Group, Aston University, Aston Triangle, Birmingham B4 7ET, United Kingdom. R. Neal and D. J. Mapps are with the School of Electronic Communications and Electrical Engineering, Faculty of Technology, University of Plymouth, Plymouth PL4 8AA, United Kingdom.

Received 26 July 2002.

0003-6935/03/193766-06\$15.00/0

(C) 2003 Optical Society of America
In general, problems can arise when one is using a LPG that is written into a conventional step-index single-mode fiber because of its sensitivity to various external influences. One problem is distinguishing between individual measurands; several papers have been published addressing this problem. ${ }^{6-8}$ Various discrimination schemes have been used to address this problem, including a pair of fiber Bragg gratings (FBGs), ${ }^{9-12}$ a LPG working in series with a FBG, ${ }^{13}$ a FBG working in conjunction with fiber Fabry-Perot devices, ${ }^{14}$ and a sampled FBG. ${ }^{15}$

We report for the first time to our knowledge the fabrication and characterization of a single LPG fiber sensor that uses information from a limited spectral region to distinguish between spectral variation caused by changes in temperature and the surrounding medium's refractive index. Therefore this LPG fiber sensor can be used for simultaneous or separate measurements of temperature and refractive index with a single optical source. We have also studied the bend sensitivity of this device. The device consists of a single grating inscribed across the junction between a bare single-mode fiber and a region coated with $\mathrm{Ag}$.

\section{Fabrication of the Long-Period Grating Sensing Device}

The fabrication of the LPG device was done in two parts. First, a length of bare B-Ge-codoped stepindex single-mode fiber was placed into a sputter machine. A section of the fiber $(4 \mathrm{~cm})$ was sputtered 


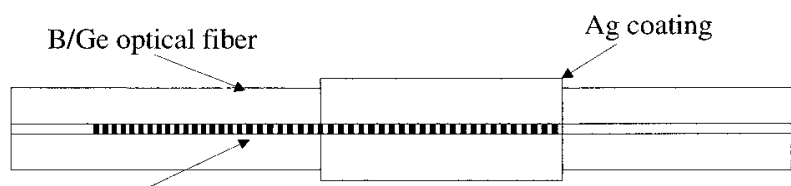

Long-period grating $=325 \mu \mathrm{m}$, length $=8 \mathrm{~cm}$

$4 \mathrm{~cm} \longrightarrow 4 \mathrm{~cm}$

Fig. 1. Schematic of the fiber LPG sensing device. with a uniform layer of Ag with a thickness of $1 \mu \mathrm{m}$. The uniform thickness of the Ag was achieved by a mechanical rotational rig placed in the sputter machine. Second, the LPG was written into the fiber with a point-by-point writing technique using a programmable shutter (a spatial period of $325 \mu \mathrm{m}$ was chosen) (see Fig. 1).

The ultraviolet beam was generated from a frequency-doubled argon-ion laser, which was focused to a size of approximately $10 \mu \mathrm{m}$. The LPG in

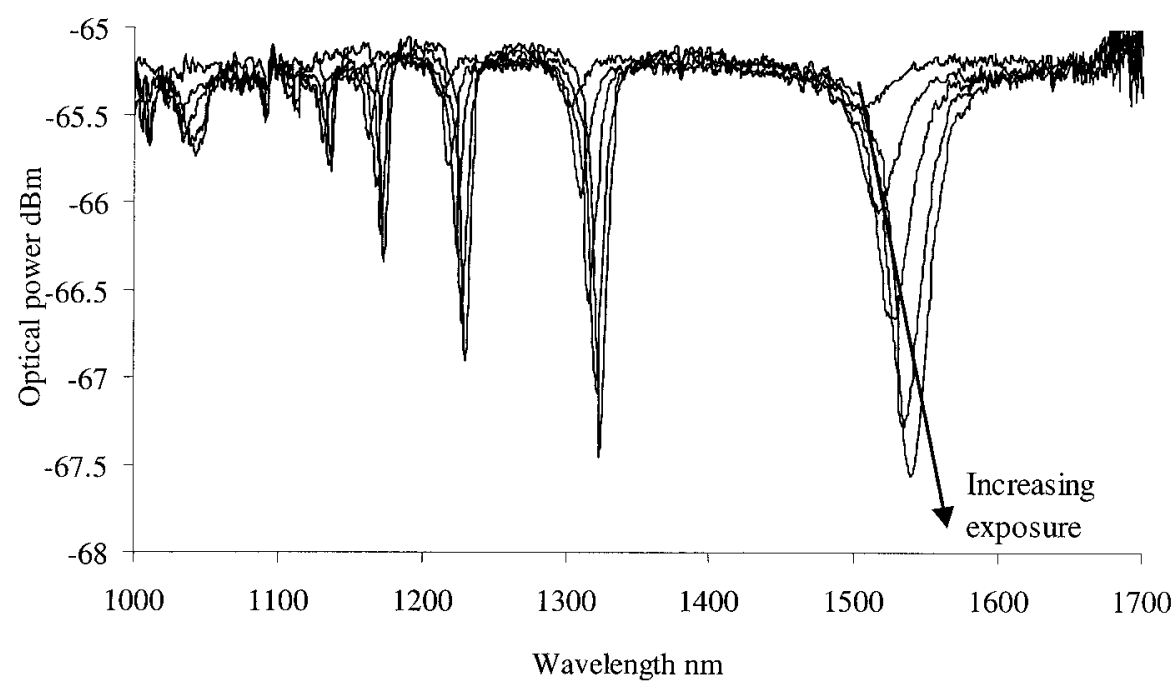

(a)

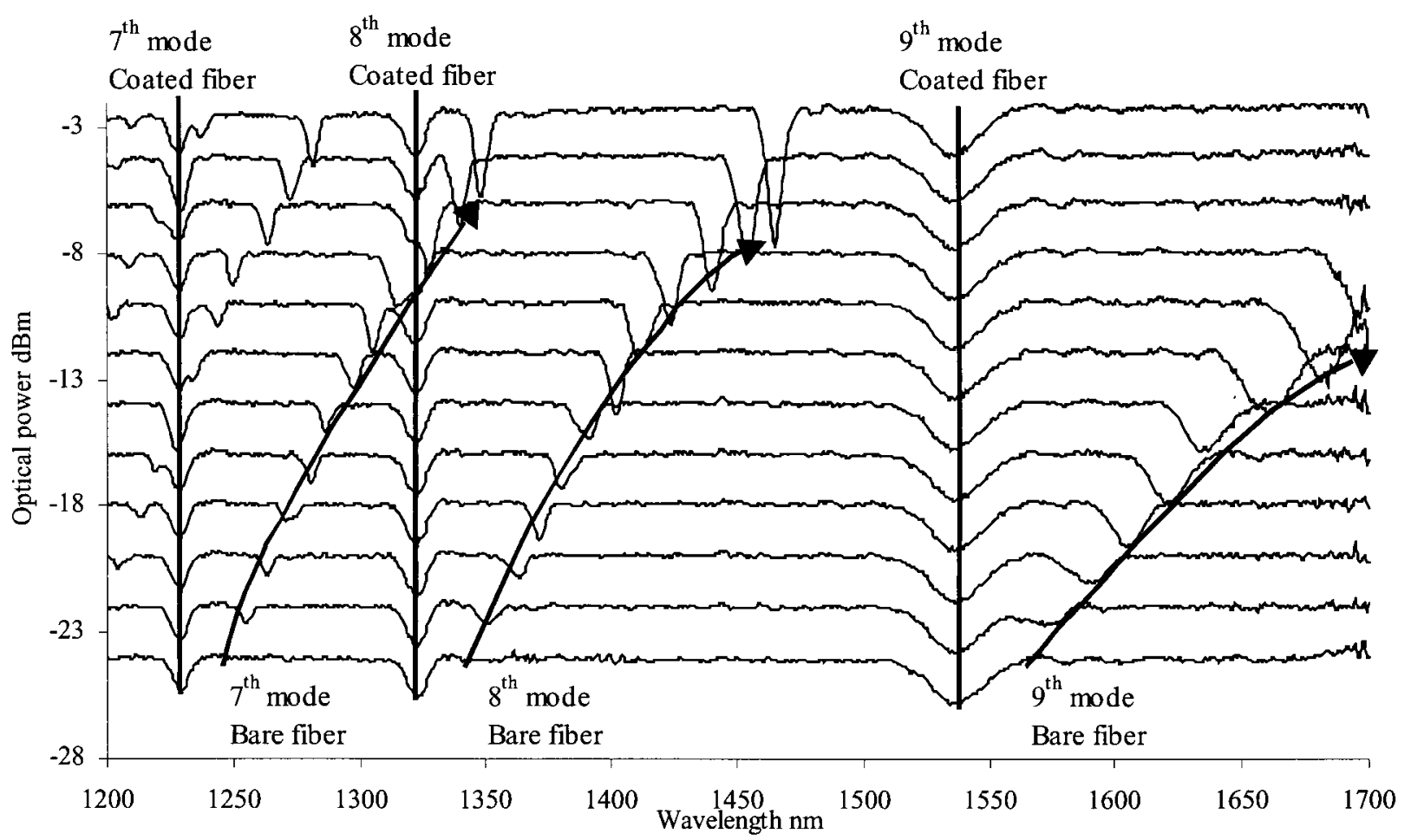

(b)

Fig. 2. (a) Transmission spectrum during fabrication of the LPG (period $=325 \mu \mathrm{m}$, length $L=4 \mathrm{~cm}$ ) in 1 - $\mu \mathrm{m}$ Ag-coated B-Ge-codoped optical fiber. (b) Transmission spectrum during the fabrication of the LPG (period $=325 \mu \mathrm{m}, L=4 \mathrm{~cm}$ ) in the bare-fiber section. Traces offset for clarity. 


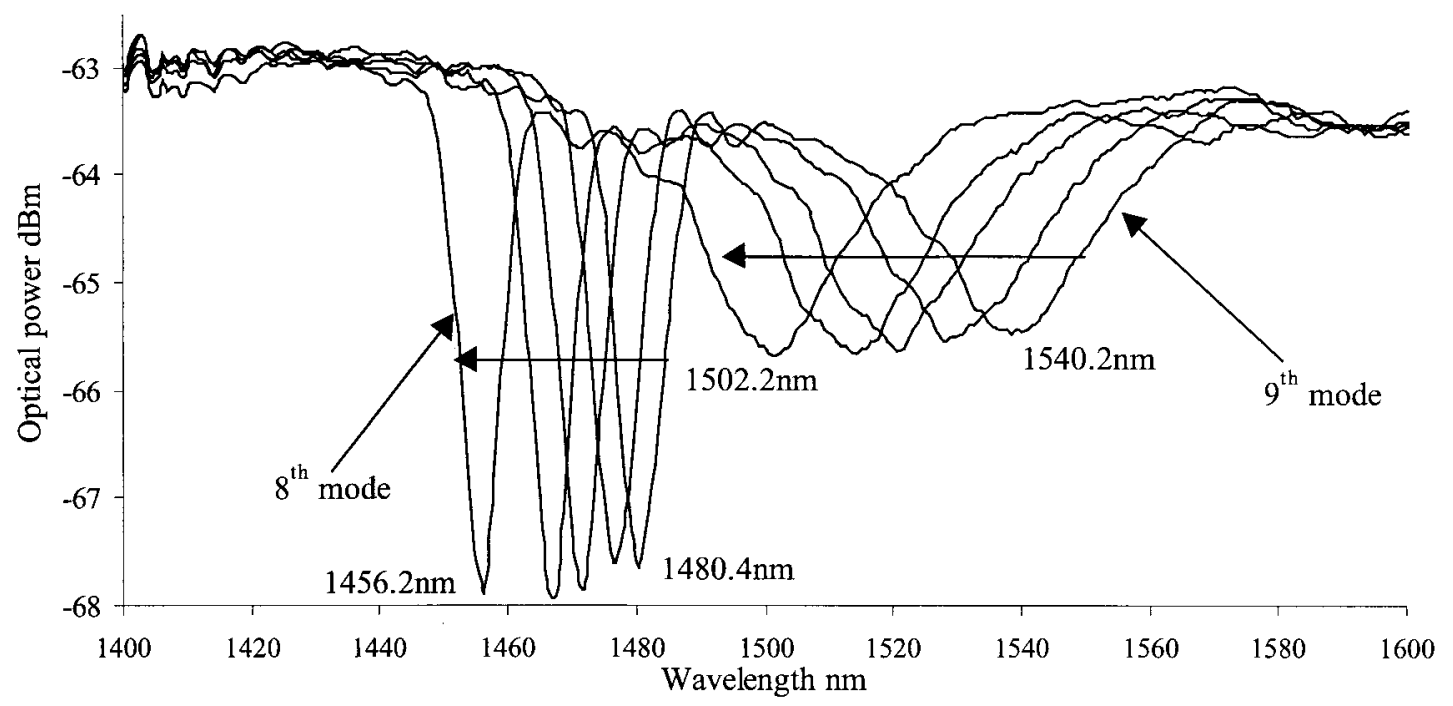

Fig. 3. Effect of temperature on the transmission spectrum of the LPG fiber device over a range $22-82{ }^{\circ} \mathrm{C}$. Arrows show the direction of increasing temperature.

the Ag-coated fiber was fabricated first, with a power of $120 \mathrm{~mW}$ at a scan rate of $0.05 \mathrm{~mm} \mathrm{~s}^{-1}$; the power used for the LPG in the bare fiber was $90 \mathrm{~mW}$ at a scan rate of $0.27 \mathrm{~mm} \mathrm{~s}^{-1}$. Throughout the recording process, the attenuation bands were monitored with an optical spectrum analyzer (OSA). Figure 2(a) illustrates the recording process in the coated fiber, with the strongest stop band $(\sim 3 \mathrm{~dB})$ occurring at a wavelength of $1540 \mathrm{~nm}$. Figure 2(b) shows the attenuation bands forming during subsequent inscription in the bare fiber.

It can be seen that, owing to the different recording characteristics of the two regions, the attenuation band of the eighth cladding mode in the bare section is spectrally located close to the attenuation band of the ninth cladding mode in the Ag-coated section $(\sim 60 \mathrm{~nm})$. This raises the interesting possibility of using a single light source to interrogate this kind of LPG device. The evolution of the attenuation bands in both sections during fabrication was expected to be different because the $\mathrm{Ag}$ coating modifies the waveguide structure. The spectral behavior of the attenuation bands reflects the behavior of the associated cladding modes, which are dependent on the material and geometry of the waveguide for their effective indices and group refractive indices. ${ }^{16}$

\section{Spectral Sensitivity of the Long-Period Grating Sensor}

\section{A. Temperature Sensitivity}

The LPG sensor was subjected to three measurands: temperature, changes in the surrounding medium's refractive index, and bending. In all cases measurements were performed by using an OSA with an internal broadband light source and with an accuracy of $0.04 \mathrm{~nm}$. The sensitivity to temperature was investigated by placing the sensor under a thermally insulating cover and on a hot plate. The tempera- ture of the hot plate was measured by using a probe with an accuracy of $\pm 0.1{ }^{\circ} \mathrm{C}$. The spectral locations of the central wavelengths of the attenuation bands associated with the eighth cladding mode in the barefiber section and the ninth cladding mode in the $\mathrm{Ag}$ coated section were monitored, and the results are shown in Fig. 3.

It can be seen from Fig. 3 that the spectral sensitivity of the two attenuation bands are dissimilar even though they are spectrally close, this is expected because each attenuation band is associated with a different cladding mode. The spectral shift of the center wavelengths of these two attenuation bands as a function of change in temperature is shown in Fig. 4.

Over the temperature range of $22-82{ }^{\circ} \mathrm{C}$ we observed a total wavelength shift of $-27.8 \mathrm{~nm}$ for the bare section and $-45.4 \mathrm{~nm}$ for the Ag-coated section, giving temperature sensitivities of $\mathrm{d} \lambda / \mathrm{d} T=0.4576 \pm$ $2 \times 10^{-4} \mathrm{~nm}^{\circ} \mathrm{C}^{-1}$ and $\mathrm{d} \lambda / \mathrm{d} T=0.7282 \pm 4 \times 10^{-4}$ $\mathrm{nm}{ }^{\circ} \mathrm{C}^{-1}$, respectively. Comparing the experimental result for the eighth mode with the results for a similar fiber described in Ref. 17 as $0.579 \mathrm{~nm}^{\circ} \mathrm{C}^{-1}$ shows a lower sensitivity, this may be due in part to the different spectral locations of the attenuation bands. The temperature resolutions of the LPG device, defined as the root-mean-square deviations from linearity, are $\pm 0.4{ }^{\circ} \mathrm{C}$ for the attenuation band associated with the bare-fiber section and $\pm 0.3^{\circ} \mathrm{C}$ for the attenuation band associated with the Ag-coated section.

\section{B. Refractive-Index Sensitivity}

The experimental apparatus used to measure the dependence of the sensor's spectral characteristics with respect to external index $n_{s}$ was the same as that used for the temperature measurement. However, this time the LPG was placed in a V groove and immersed in certified refractive-index (CRI) liquids 


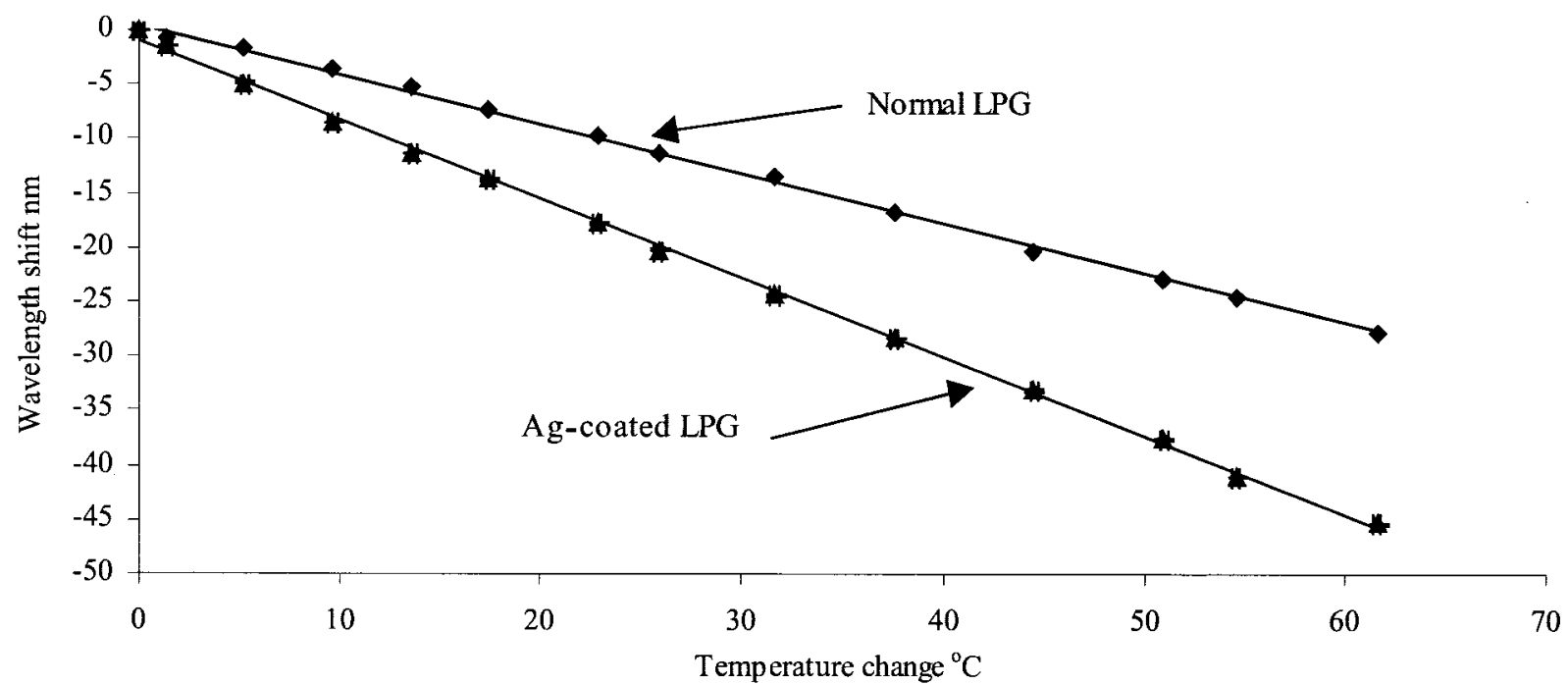

Fig. 4. Spectral sensitivity of the two attenuation bands associated with each section of the LPG fiber device as a function of temperature change. Linear fits to the points are shown.

(supplied by Cargille Laboratories, Inc.) that have a quoted accuracy of \pm 0.0002 .

The LPG and V groove were carefully cleaned, washed in methanol, then in deionized water, and finally air dried before the immersion of the LPG into a CRI liquid ranging from $n_{s}=1.300$ to $n_{s}=1.452$. This cleaning procedure was adopted to minimize the amount of salts deposited on the fiber by the chemicals. The $\mathrm{V}$ groove was made in an $\mathrm{Al}$ plate and machined flat to reduce bending of the fiber. The plate was placed on an optical table, which acted as a heat sink to maintain a constant temperature. This was done to minimize thermally induced spectral drift of the attenuation bands. The results are shown in Figs. 5 and 6.
Figure 5 shows that the attenuation band associated with the Ag-coated section is essentially unaffected by $n_{s}$. This was confirmed by immersing the LPG device into a CRI liquid with a refractive index $n_{s}=1.70$, which again produced no discernible shift. This was to be expected, since the penetration depth of $\mathrm{Ag}$ at a wavelength of $\sim 1500 \mathrm{~nm}$ is $\sim 10 \mathrm{~nm} .^{18}$ Therefore the E field of the cladding mode associated with the attenuation band in the Ag-coated section would be significantly attenuated in the $1-\mu \mathrm{m}$ thickness of Ag, so the $n_{s}$ has negligible effect. Note, however, that the penetration depth in the region from $\sim 230$ to $\sim 300 \mathrm{~nm}$ varies from $\sim 20$ to $\sim 0.1 \mu \mathrm{m}$, making it possible to fabricate the LPG through the $1-\mu \mathrm{m}$ Ag coating with a long exposure.

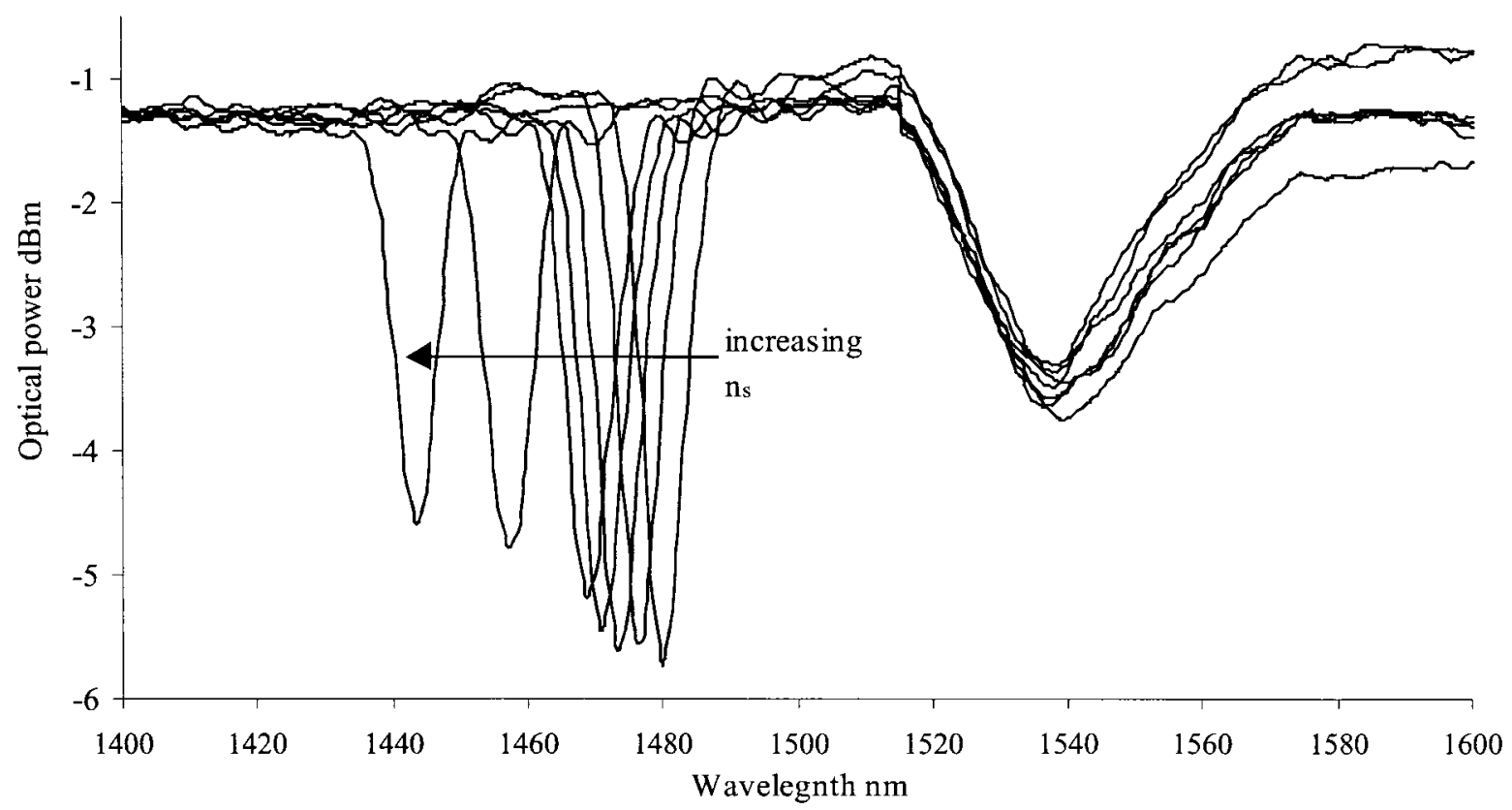

Fig. 5. Effect of increasing refractive index of the surrounding medium on the transmission spectrum of the LPG fiber device. 


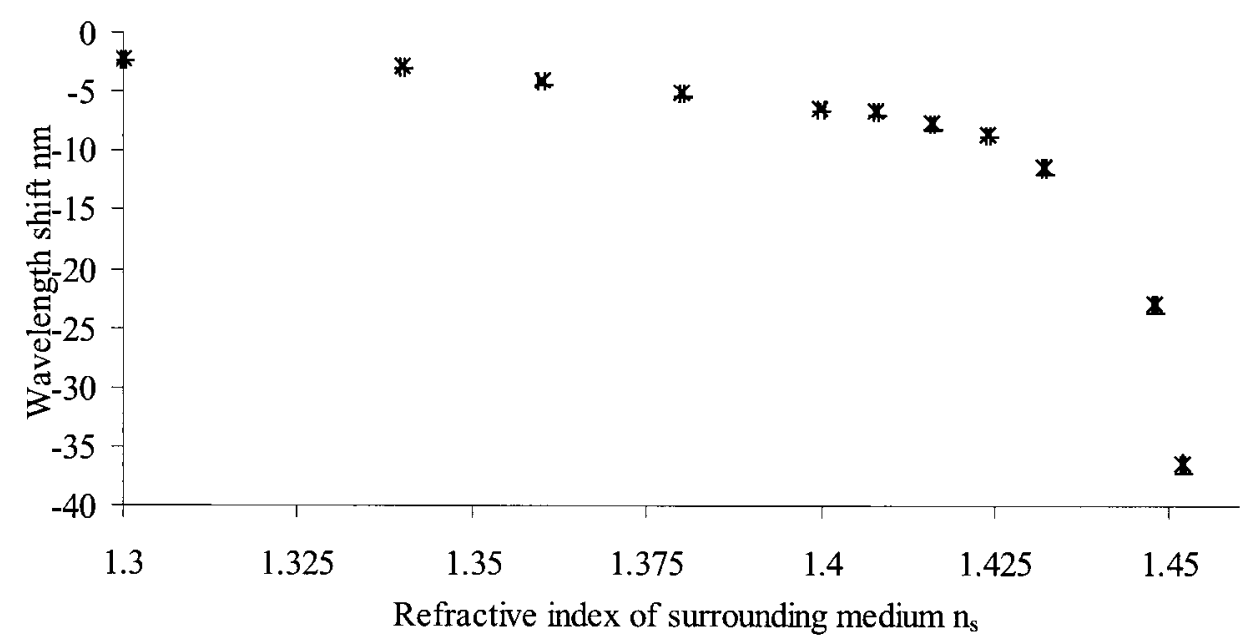

Fig. 6. Experimental spectral sensitivity of the attenuation band associated with the bare-fiber section of the LPG fiber device as a function of $n_{s}$.

The attenuation band associated with the barefiber section demonstrates the usual spectral characteristics of a LPG with regard to $n_{s}$. This is shown in Fig. 6.

The linear-regression technique was employed on several sets of experimental data to determine the spectral response of the LPG device to changes in the surrounding medium's refractive index, which is indicated by the error bars in Fig. 6. The deviation in the experimental results yields a wavelength resolution of $\sim \pm 0.066 \mathrm{~nm}$ for the eighth mode for the attenuation band associated with the bare fiber around a refractive index of 1.375. This translates to approximate refractive-index resolutions $\left(\Delta n_{s}\right)$ of $\pm 9.6 \times 10^{-4}$ in that region.

\section{Bending Sensitivity}

The third investigation was to obtain the sensor's spectral sensitivity to bending. This was achieved with the experimental setup shown in Fig. 7.

The LPG device was clamped between two towers; one of the clamps was mounted on a translation stage that was moved inward to induce a bend in the optical fiber. For this arrangement with the LPG midway between the clamps, the sensor's curvature, $R$, is given in Ref. 19 as

$$
R=\frac{2 / d}{d^{2}+L^{2}}
$$

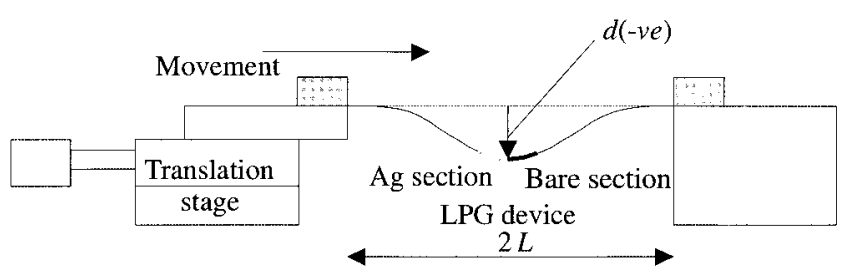

Fig. 7. Schematic of the bending apparatus. where $L$ is the half-distance between the edges of the two towers and $d$ is the bending displacement at the center of the LPG. The central wavelength of the two attenuation bands was monitored with an OSA. The results are shown in Fig. 8.

It can be seen from Fig. 8 that the wavelength shifts of both attenuation bands are not a linear function of curvature; a quadratic gives a reasonable fit to the experimental data, as shown in the figure. The departure of the curves from the fitted polynomial correspond to a wavelength resolution of $\sim \pm 0.2 \mathrm{~nm}$ for the eighth mode and $\sim \pm 0.3 \mathrm{~nm}$ for the ninth mode, which translate to approximate curvature resolutions of $\sim \pm 0.037$ and $\sim \pm 0.04 \mathrm{~m}^{-1}$, respectively.

It should be pointed out that the calculation of the curvature assumes that the fiber has a uniform cross section. In our case, the Ag-coated section will have slightly higher rigidity than the bare fiber and hence will have a slightly lower curvature. The larger values of wavelength shift exhibited by the coated section are a result of the attenuation band's being associated with a higher-order cladding mode, which leads in this case to a greater sensitivity.

\section{Conclusion}

We have described the fabrication and characterization of a temperature self-compensating single LPG fiber device based on a two-section LPG, with one section of the fiber being Ag-coated before its fabrication. The strength of this Ag-coated stop band after fabrication was $\sim 3 \mathrm{~dB}$. The attenuation band associated with the Ag-coated fiber is unaffected by changes in the surrounding medium's refractive index, which was tested up to $n_{s}=1.7$, and has a temperature sensitivity of $-0.728 \mathrm{~nm}^{\circ} \mathrm{C}^{-1}$. Thus it can be used to compensate for temperature effects. The scheme used to interrogate this sensor yields resolutions of $\pm 1.0 \times 10^{-3}$ for a refractive index in the region of 1.375 and $\pm 0.3^{\circ} \mathrm{C}$ for temperature.

This property is due to the penetration depth of $\mathrm{Ag}$ 


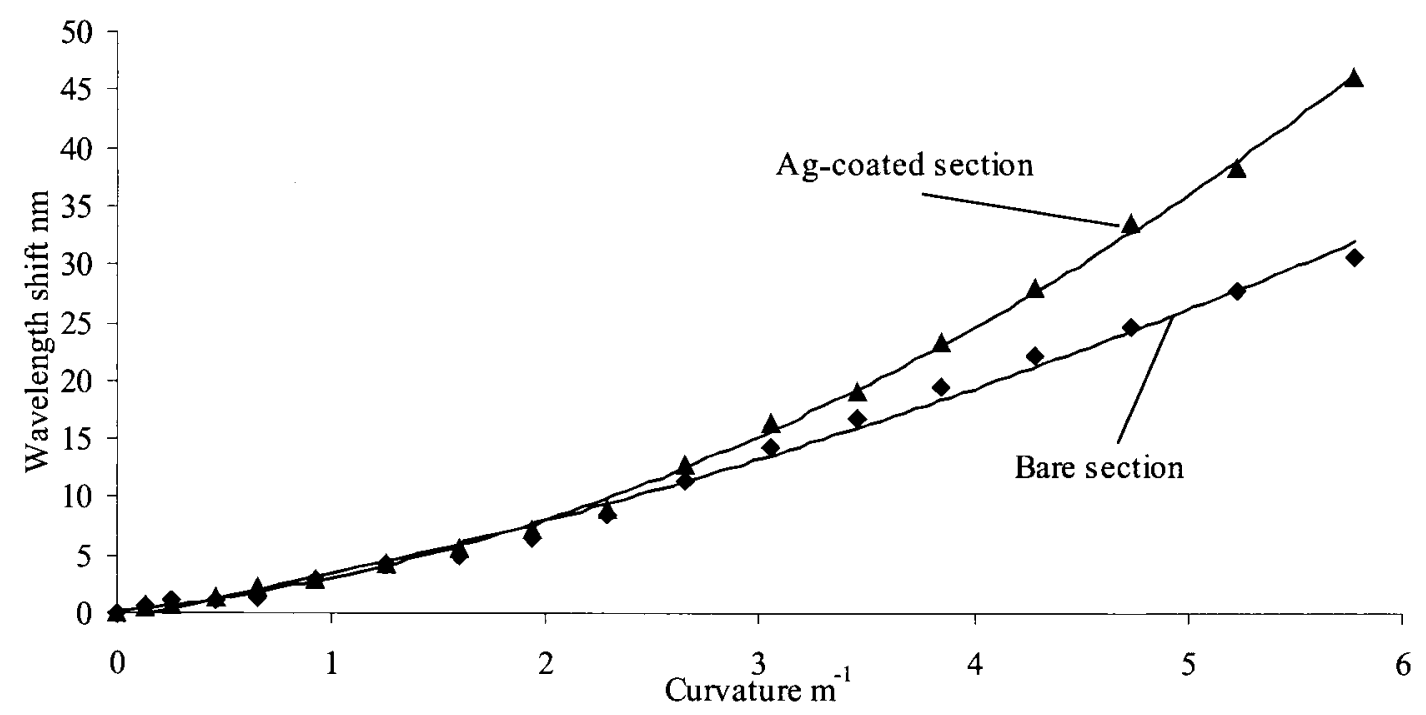

Fig. 8. Experimental central wavelength shift of the attenuation bands as a function of curvature subjected to the LPG device.

at a wavelength of $1500 \mathrm{~nm}$, which is $\sim 20 \mathrm{~nm}$. Also, the spectral response of the two attenuation bands to bending was investigated, and it was found that there was a significant difference between the attenuation bands, with the Ag-coated section's stop band having a greater sensitivity at curvatures in excess of $3 \mathrm{~m}^{-1}$.

This type of LPG may be used to overcome the recoating problems caused by the sensitivity to external refractive index of normal LPGs in step-index fibers that are used for gain flattening in the telecommunications industry. Also, this sensor can be used for simultaneous measurement of temperature and the refractive index of the surrounding medium. Finally, the attenuation bands are spectrally close to each other, allowing a single light source to be used to interrogate the device, thus reducing costs.

\section{References}

1. Y. Ruan, X. Huang, Y. Jiang, and J. Wang, "The design and simulation of cascade long-period fiber gratings for flattening EDFA's gain," in Photorefractive Fiber and Crystal Devices: Materials, Optical Properties and Applications V, F. T. Yu, and S. Yin, eds., Proc. SPIE 3801, 240-245 (1999).

2. C. D. Su and L. A. Wang, "Linewidth broadening of Er-doped superfluorescent fiber source using long-period grating," Electron. Lett. 35, 331-332 (1999).

3. A. Vengsarkar, P. Lemaire, J. Judkins, V. Bhatia, T. Erdogan, and J. Sipe, "Long-period gratings as band-rejection filters," J. Lightwave Technol. 14, 58-64 (1996).

4. V. Bhatia and A. M. Vengsarkar, "Optical fiber long-period grating sensors," Opt. Lett. 21, 692-694 (1996).

5. H. J. Patrick, A. D. Kersey, and F. Bucholtz, "Analysis of the response of long period fiber gratings to external index of refraction," J. Lightwave Technol. 16, 1606-1612 (1998).

6. V. Grubsky and J. Feinberg, "Long-period gratings with variable coupling for real-time sensing applications," Opt. Lett. 25, 203-205 (2000).

7. V. Bhatia, T. D'Alberto, N. Zabaronick, and R. Claus, "Temperature-insensitive and strain-insensitive long-period gratings sensor for smart structures," Opt. Eng. 36, 1872-1875 (1997).

8. R. P. Espindola, R. Windeler, A. Abramov, B. Eggleton, T.
Strasser, and D. DiGiovanni, "External refractive index insensitive air-clad long period fibre grating," Electron. Lett. 35, 327-328 (1999).

9. W. James, M. L. Dockney, and R. P. Tatam, "Simultaneous independent temperature and strain measurement using infibre Bragg grating sensors," Electron. Lett. 32, 1133-1134 (1996).

10. M. G. Xu, J.-L. Archambault, L. Reekie, and J. P. Dakin, "Discrimination between strain and temperature effects using dual-wavelength fibre grating sensors," Electron. Lett. 30, 1085-1087 (1994).

11. M. Song, S. Lee, S. Choi, and B. Lee, "Simultaneous measurement temperature and strain using two fiber Bragg gratings embedded in a glass tube," Opt. Fiber Technol. Mater. Devices Syst. 3, 194-196 (1997).

12. B. O. Guan, H. Y. Tam, S. L. Ho, W.-H. Chung, and X. Y. Dong, "Simultaneous strain and temperature measurement using a single fibre Bragg grating," Electron. Lett. 36, 1018-1019 (2000).

13. H. Patrick, G. Williams, A. Kersey, J. Pedrazzani, and A. Vengsarkar, "Hybrid fiber Bragg grating/long period fiber grating sensor for strain/temperature discrimination," IEEE Photon. Technol. Lett. 8, 1223-1225 (1996).

14. Y. J. Rao, P. J. Henderson, D. A. Jackson, L. Zhang, and I. Bennion, "Simultaneous strain, temperature and vibration measurement using a multiplexed in-fibre-Bragg-grating/fibreFabry-Perot sensor system," Electron. Lett. 33, 385-387 (1997).

15. X. Shu, B. Gwandu, Y. Liu, L. Zhang, and I. Bennion, "Sampled fiber Bragg grating for simultaneous refractive index and temperature measurement," Opt. Lett. 26, 774- 776 (2001).

16. T. Allsop, L. Zhang, and I. Bennion, "Detection of organic aromatic compounds in paraffin by a long period fibre grating optical sensor with optimised sensitivity," Opt. Commun. 191, 181-190 (2001)

17. X. Shu, T. Allsop, B. Gwandu, L. Zhang, and I. Bennion, "Hightemperature sensitivity of long period gratings in B-Ge codoped fiber," IEEE Photon. Technol. Lett. 13, 818-820 (2001).

18. M. Born and E. Wolf, Principles of Optics, 6th ed. (Pergamon, Exeter, 1980).

19. W. Du, H. Tam, M. Liu, and X. Tao, "Long-period fiber grating bending sensors in laminated composite structures," in Sensory Phenomena and Measurement Instrumentation for Smart Structures and Materials, R. O. Claus and W. B. Spillman, eds., Proc. SPIE 3330, 284-292 (1998). 